\title{
Analysis of Logistics Service Quality and Customer Satisfaction during COVID-19 Pandemic in Saudi Arabia
}

\author{
Amjaad Bahamdain ${ }^{1}$, Zahyah H. Alharbi ${ }^{2}$, Muna M. Alhammad ${ }^{3}$, Tahani Alqurashi ${ }^{4}$ \\ Management Information Systems Department, King Saud University, Riyadh, Saudi Arabia ${ }^{1,2,3}$ \\ Common First Year Deanship, Computer Science Department, Umm Al-Qura University, Makkah, Saudi Arabia ${ }^{4}$
}

\begin{abstract}
Logistics companies' success is inextricably linked to the quality of their services, particularly when dealing with customer issues. Nowadays, social media is the first place that users turn to in order to express their thoughts on services or to communicate with customer service representatives to resolve problems. Businesses can retrieve and analyze these data to gain a better understanding of the factors that affect their operations, both positively and negatively. During the COVID-19 pandemic, we conducted a sentiment analysis to assess customer satisfaction with logistics services in Saudi Arabia's private and public sectors. Using a lexicon-based approach, 67,124 tweets were collected and classified as positive, negative, or neutral. A support vector machine (SVM) model was used for classification, with an average accuracy of $82 \%$. Following that, we conducted a thematic analysis of negative opinions in order to identify the factors that influenced the effectiveness and quality of logistics services. The findings reveal five negative themes: delay, customer service issues, damaged shipments, delivery issues, and hidden prices. Finally, we make suggestions to improve the efficiency and quality of logistics services.
\end{abstract}

Keywords-Logistics services; sentiment analysis; lexiconbased approach; SVM; sentiment classification

\section{INTRODUCTION}

Logistics adds value by meeting customers' delivery needs in a cost-effective manner [1]. As a result, logistics service performance represents a provider's ability to consistently deliver requested products within the specified time frame and at an acceptable cost [2, p. 34]. This means that the quality of the provided logistics services can be considered the most significant success guarantor of a logistics business [3]. It increases customer satisfaction, which in turn increases the business's profitability and competitive advantage [4]. Customer satisfaction will determine whether or not customers make further purchases or refer the business to others [5].

People rely heavily on social media for information, news, and entertainment. Twitter and other social media platforms are growing in popularity because they provide easy access to real-time posts on a daily basis. Tweets and mentions on Twitter contain information about current events, news, user opinions, and reviews. These data are useful to businesses because they allow them to better understand user preferences, improve their services, and increase customer satisfaction. Twitter provides an Application Programming Interface (API) through which researchers, developers, and business owners can obtain data to analyze and learn more about topics of interest to them. Sentiment Analysis is the process of extracting, analyzing, and distinguishing opinions or emotions from text [6]. It is a branch of natural language processing (NLP) research. Sentiment analysis is currently used to analyze data posted on social media platforms or websites in order to determine opinions, attitudes, or emotions about businesses, products, or services. These classifications are beneficial to business owners because they allow them to identify their strengths and weaknesses in order to improve future services and thus increase profits. To the best of our knowledge, no previous studies have used Twitter users' opinions to analyze customer satisfaction with logistics services in Saudi Arabia during the COVID-19 pandemic. Therefore, this study aims to fill this gap and to achieve the following objectives: 1) to investigate customer satisfaction with logistics services during COVID-19 in Saudi Arabia by analyzing customer opinions; and 2) to identify the elements of logistics service quality that influence customers' sentiments toward logistics service providers.

The following is how the paper is structured. First, we review the relevant literature, and describe the methods employed to collect the data and conduct the analysis. The results are presented, followed by a discussion of the results. Finally, the managerial implications of the findings and future research directions are explored.

\section{LiterATURE REVIEW}

This section begins by reviewing logistics service quality and customer satisfaction. Second, we discuss the various tools, methods, and results of sentiment analysis studies that have used Arabic tweets. We also review how researchers have used sentiment analysis to investigate customer satisfaction.

\section{A. Logistics Service Quality and Customer Satisfaction}

Customer satisfaction is defined as the perceived difference between prior expectations and actual product or service performance [7]. It is a sign that customers' needs are being met in a pleasurable manner. According to some researchers, the ultimate goal of any organization is to meet the needs and achieve the satisfaction of its customers. Understanding the needs and expectations of customers is a necessary step in ensuring their satisfaction [8]. 
The research of Parasuraman, Zeithaml, and Berry [9] identified five broad dimensions of service quality that influence customer satisfaction: dependability, receptiveness, assurance, empathy, and tangibles. Dependability explains the ability to provide the promised service consistently and reliably. Receptiveness represents the willingness to assist customers and provide prompt service. Assurance symbolizes employee knowledge and courtesy, as well as the ability to convey trust and confidence, while empathy is about providing caring and individualized attention to customers. Finally, tangibles represent the appearance of physical facilities, equipment, personnel, and communications materials. Several studies have applied these dimensions to identify the quality gap in logistics and to improve the services in order to achieve customer satisfaction. For example, [10] conducted a study to validate five determinants of service quality and to investigate the service quality-customer satisfaction link in the port logistics service industry in Vietnam. According to the findings, five factors influence the quality of port logistics services: responsiveness, assurance, dependability, tangibles, and empathy. The findings additionally show that the quality of port logistics services has a positive impact on customer satisfaction.

Furthermore, Cho et al. [11] presented three factors of service quality in order to determine their effects on customer satisfaction, referral intentions, and loyalty. The study was linked to the port logistics service industry in Incheon and Shanghai. The result of their study showed that exogenous and relational quality remained important dimensions of customer satisfaction. According to their findings, relational quality included the positive attitude and professionalism of employees, partnership relations between the customers and the ports, and the ability of the port information system to provide timely information. However, the endogenous quality of services has an insignificant impact on customer satisfaction.

Uvet [12] conducted a study that investigated the aspects of logistics services that affected customer satisfaction. These included timeliness, quality of personal contact, operational information sharing, order condition and handling of order discrepancies. He applied structural equation modeling (SEM) and confirmatory factor analysis (CFA) to examine customer satisfaction by utilizing the five factors of logistics service quality. The results showed that there are significant relationships between logistics service quality factors and customer satisfaction. Additionally, the finding of this study can be applied in any business to obtain competitiveness in logistics services.

\section{B. Sentiment Analysis and Customer Satisfaction}

Anastasia and Budi [13] examined Twitter opinion data to estimate users' satisfaction with the GO-JEK and Grab companies, which provide online transportation services in Indonesia. From February to March 2016, they retrieved 126,405 tweets using the Twitter API and the R programming language. RapidMiner was also used for data pre-processing and classification. The study examined the highest accuracy score using three classification algorithms: SVM, Naïve Bayes
(NB), and Decision Tree. The results showed that Grab had higher user satisfaction than GO-JEK, and that the SVM and Decision Tree algorithms had the highest accuracy with a score of $72.97 \%$.

From September 2013 to February 2014, Gitto and Mancuso [14] conducted an exploratory sentiment analysis on passenger review data extracted from a blog called "Airport Reviews” on the SKYTRAX website. Their study looked at customer satisfaction for both aviation and non-aviation airport services. The study looked at five international airports in Europe: Amsterdam Schiphol, Frankfurt, London Heathrow, Madrid Barja, and Paris Charles de Gaulle. The researchers used KNIME, an open-source platform for data analytics, and Semantria, which uses a dictionary base and a machine learning technique, to conduct sentiment analysis and to detect positive, neutral, or negative sentiments. According to the findings, non-aviation services such as food and beverage and the shopping area had the greatest influence on passenger satisfaction levels. Non-aviation services received approximately 55\% positive feedback, while aviation services received only $33 \%$.

Bhattacharjya et al. [15] investigated the effectiveness of customer services on Twitter for logistics services provided by e-retailers. The study looked at conversations between customers and businesses rather than individual tweets to investigate the effectiveness of customer services. A large sample of 203,349 tweets from e-retailers was collected, as well as a random sample of logistics conversations. After data preparation and cleansing, a total of 16,998 tweets were analyzed. According to the findings, e-retailers and logistics service providers mostly redirect customers to other channels to solve their problems, while ignoring their customers' needs to find solutions and get their issues resolved on Twitter. The findings also revealed a lack of interaction between e-retailers and logistics service providers over providing effective customer service.

Ahmadi et al. [16] performed a sentiment analysis on public service conversations in a hospital in Yogyakarta, Indonesia. The study created the "Kata Kita" product to translate conversations into text and to help deaf people communicate with service personnel by displaying the text on the screen. Customer satisfaction was also evaluated. The study combined NLP with K-nearest neighbors (KNN) and the term frequency-inverse document frequency (TF-IDF) algorithm to classify conversations as "satisfied" or “dissatisfied”. The results showed $74.00 \%$ accuracy, $76.00 \%$ precision, and $73.08 \%$ recall.

This literature review demonstrates that researchers all over the world have been focusing on customer opinions in order to better understand customers and the factors that influence their satisfaction. Furthermore, due to the significance of this type of analysis and the scarcity of studies using sentiment analysis to analyze Arabic tweets to understand customers' satisfaction with logistics services, this study will analyze customers' opinions of logistics services during the COVID-19 pandemic. 


\section{Arabic Sentiment Analysis}

Several studies have used Arabic sentiment analysis to gain a better understanding of various phenomena. Aldayel and Azmi [17], for example, proposed a hybrid approach that combined a lexicon-based technique with machine learning techniques to develop a sentiment analysis of Arabic tweets retrieved via Twitter's API that addressed social issues in Saudi Arabia. They used data cleaning, normalization, stopword removal, an n-gram model to remove repeated letters, and a light stemmer for Arabic text stemming during the preprocessing phase. The training data were labeled using a lexicon-based classifier, and the classification model was built using an SVM classifier. According to the study's findings, the hybrid approach achieved an F-measure of $84 \%$ and an accuracy of $84.01 \%$.

Al-Ghaith [18] created the SaudiSentiPlus, a Saudi dialect lexicon that includes a translation of English sentiment lexicons as well as 7,139 terms manually extracted from Saudi tweets. The dataset's polarity classification was carried out manually by three annotators who were Arabic native speakers and Saudis. They divided the dataset into three categories: positive, negative, and neutral. For each classification, the output was 300 labeled tweets. The study conducted an experiment to assess the performance of the SaudiSentiPlus lexicon, using 971,000 tweets from Saudi dialect hashtags as a testing dataset. The accuracy of their results was $81 \%$, which is considered very good.

Al-Horaibi and Khan [19] proposed a sentiment classification system based on a combination of Arabic and English sentiment lexicons. A total of 14,984 tweets were collected from Twitter using the Twitter API and the Python Tweepy2 library. The tweets were pre-processed by the researchers, who removed @usernames, hashtag signs, symbols, numbers, non-Arabic letters, and re-tweets. Following the cleaning process, a total of 3,200 tweets were produced, and these were annotated by two native Arabic annotators. The researchers only used 2,000 tweets because the remaining tweets created uncertainty. There were 634 positive tweets, 675 negative tweets, 691 neutral tweets, and 26,349 tokens in total. A classification semantic approach that used the Arabic Sentiment Lexicon (ArSneL) and the English SentiWordNet lexicon was then employed. The findings indicated that there was a need to improve Arabic stemmer tools by including more roots to support the search process. In addition, the Arabic spelling correction tools needed to be improved, and more words needed to be added.

Al-Hussaini and Al-Dossari [20] proposed a sentiment analysis approach based on a lexicon that included a Saudi dialect lexicon. The lexicon was built using a semiautomatic building technique, with the lexicon being built manually after the data were collected from Twitter. Then, two lists were manually constructed: one with the most frequently occurring positive sentiment words; and the other with the most frequently occurring negative sentiment words. The lexicon was divided into two columns: words and their polarities. Following classification, there were 762 positive words/phrases with a +1 value and 662 negative words/phrases with a -1 value. The results show that the developed Saudi dialect lexicon helped to improve the sentiment labeling of Arabic tweets.

\section{RESEARCH METHODOLOGY}

In this section, we will present our Arabic sentiment analysis methodology, which consists of four phases: data collection, data pre-processing, lexicon-based classification, and sentiment analysis. Fig. 1 illustrates the implemented Arabic sentiment analysis methodology, and the following sections explain each stage in more detail:

\section{A. Data Collection Phase}

The Python Tweepy2 library was used to extract tweets from the Twitter API. In total, 67,124 tweets were collected that mentioned public and private logistics companies. These included Aramex, DHL, Fetchr, Naqel, SMSA, and Saudi Post. The retrieved tweets were tweeted during the surge of the COVID-19 pandemic, precisely from June to September 2020.

\section{B. Data Pre-processing Phase}

The data pre-processing phase consists of three successive stages: text cleaning, tokenization and stemming. In the text cleaning, a number of steps were performed as follows:

- Removing irrelevant tweets that includes ads and were not related to our task.

- Removing non-Arabic letters, including numbers, symbols, the hashtag sign and emojis as they are not part of the analysis.

- Replacing “@username”, “www.” and “https?://” with empty strings.

- Removing the punctuation.

- Eliminating character repetition in a word by replacing it with a single character, such as "ويييييين شحنتنتني" which is replaced with “وين شحنتي".

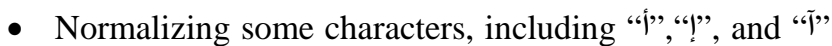

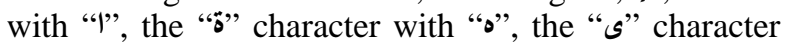

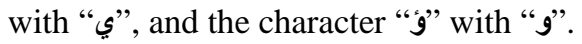

- Removing stop words, the standard Arabic stop words, provided by the Natural Language toolkit (NLTK) library, were used. 


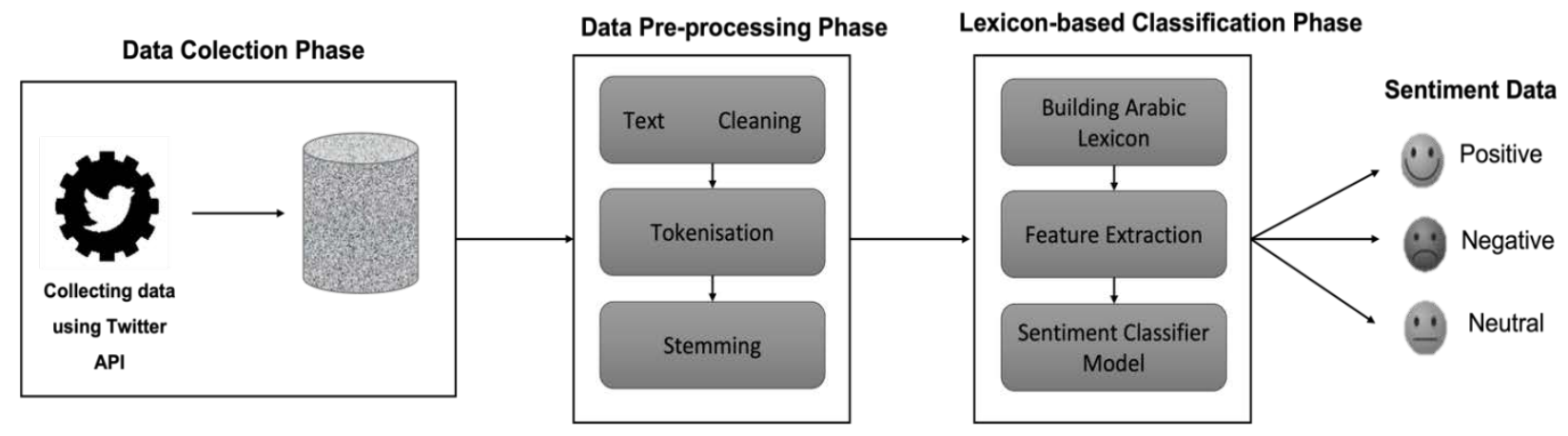

Fig. 1. The Implemented Arabic Sentiment Analysis Methodology.

Then, we split the text into tokens or words using NLTK tokenization ${ }^{1}$.

Finally, for the stemming step, we used an Arabic light stemmer called ISRI Arabic Stemmer [21] to remove prefixes and suffixes in order to obtain the root of words. The output of the stemmed words, however, was rooted into three-character words, making the majority of the words unreadable or without meaning. As a result, we did not proceed with the stemming step. According to Albraheem and Al-Khalifa [22], stemming may be ineffective for Arabic dialects as most words lack roots or have roots with more than three characters. Another study [23] confirmed that Arabic dialect does not receive adequate processing in Arabic sentiment analysis because Arabic dialectal varieties necessitate advanced pre-processing and lexicon-building steps.

\section{Lexicon-based Classification Phase}

For sentiment analysis, a lexicon-based approach was used. First, an Arabic lexicon was needed to label the data before applying the classifier algorithm. There are few Arabic lexicons available, and it is difficult to find one that includes Saudi dialects. Only two studies, [24], [25], were discovered that constructed the Arabic lexicon with Saudi dialects in mind. Therefore, we updated the lexicon created by [18] for categorizing tweets as "positive" or "negative" by including words related to logistics services in each list. The resulted lexicon contained approximately 6,500 words. The lexicon was then applied to assign polarity scores to each word in the tweets: either 1,0 , or -1 according to whether they were positive, neutral, or negative, respectively. Following that, each tweet was labeled based on its maximum polarity scores, and when a tie occurred it was labeled as neutral. For instance, if a tweet contained three negative and two positive words, it was labeled as negative, and vice versa. It was otherwise labeled as neutral.

Second, the feature extraction step was performed on our dataset. This step aims to extract numerical features from text data to make it more suitable for a classification algorithm. We used the TF-IDF algorithm, which increases the weight of rare words, while diminishing the weight of words that occur frequently in the dataset.

${ }^{1}$ NLTK (Natural Language toolkit) tokenization is the process of dividing large text into smaller parts called tokens.
After that, SVM was used to build the sentiment classifier model. SVM makes use of a learning algorithm that is based on statistical learning theory and optimization theory. It enables the computer to learn how to perform classification and regression tasks, to improve prediction accuracy, and to avoid the drawbacks of overfitting [26]. The main idea behind SVM is to find the hyper-plane that divides document vectors into two distinct classes [27]. According to several previous studies, SVM performs well with Arabic text. For example, [28] tested three different classification algorithms, the NB, SVM and KNN classifiers, and they found that the SVM classifier outperformed the other classifiers in terms of the precision measure. Another study confirming the ability of SVM to classify Arabic tweets was [29]. The authors tested six different classification algorithms: SVM, KNN, NB, Neural Network, and two Decision Tree algorithms including J48 and C5.0. They concluded that SVM outperformed the other tested classifiers. Moreover, they showed that using the SVM classifier without any stemming on Arabic text was better than other stemming methods [23]. Therefore, it was deemed to be suitable for this research.

\section{Experimental Setup}

We used the train test split () function from the Python library SciKit-learn to split the dataset into $70 \%$ for training and $30 \%$ for testing during the training phase. The data were then subjected to a 10-fold cross-validation to ensure their accuracy and to prevent overfitting. The data were divided into ten parts in the cross-validation method. The first run included nine parts for training and one for testing. Another part was used for testing in the next run, while the remaining nine parts were used for training. The final accuracy is the mean of the accuracies obtained from the ten runs. The SVM classification model then predicted the sentiments of the tweets using the predict function to put the classification model to the test.

\section{SVM PERFORMANCE EVALUATION}

To assess the performance of the SVM classifier, we employed a variety of evaluation metrics that can fairly judge the model and assess its performance. Accuracy, recall, and precision were calculated and Table I presents these results for SVM while Table II shows classification performance by sentiment polarity. The average accuracy of the SVM classifier in this study was $82 \%$. The results show that SVM has a superior ability in 3-class classification because it can distinguish among classes of sentiment polarity. 
TABLE I. RESULT OF SVM MODEL

\begin{tabular}{|l|l|l|l|l|}
\hline Classifier & Accuracy & Precision & Recall & F1 \\
\hline SVM & 0.82 & 0.81 & 0.80 & 0.80 \\
\hline
\end{tabular}

TABLE II. RESULTS OF CLASSIFICATION PERFORMANCE BY SENTIMENT POLARITY

\begin{tabular}{|l|l|l|l|l|}
\hline Classifier & Polarity & Precision & Recall & F1 \\
\hline \multirow{3}{*}{ SVM } & Neutral & 0.74 & 0.73 & 0.73 \\
\cline { 2 - 5 } & Positive & 0.82 & 0.80 & 0.81 \\
\cline { 2 - 5 } & Negative & 0.86 & 0.88 & 0.87 \\
\hline
\end{tabular}

\section{Thematic Analysis Results}

After identifying positive and negative tweets, we conducted a thematic analysis of negative opinions in order to identify the factors influencing the quality of logistics services. We used Python to search for codes in the tweets and to then identify the most frequently occurring themes. As a result, five negative opinion theme categories emerged. These themes are: customer support issues, damaged shipments, delay, delivery issues, and hidden prices. Table III displays the negative themes, along with the percentage of occurrences in the entire dataset.

\section{A. Customer Support Issues}

Customers reported a variety of problems related to customer support, including a lack of support from customer service representatives and poor service from delivery drivers. For example, $5.7 \%$ of the tweets were associated with bad customer service. Many customers complained that customer service representatives were unable or unwilling to resolve their issues - some of the linked tweets are given below (note: translated from Arabic):

"I want to file a complaint about your company, I have been waiting for my shipment for two months and I contacted you and you replied to me, we are working to follow up the request, and I did not see anything.”

"Unfortunately, I submitted a complaint without any concern and care from you."

"I filed a complaint and the matter was neglected as if it was an unimportant report and they did not contact me till now."

"I sent complaints till I got bored, yet no one answers."

TABLE III. NEGATIVE THEMES

\begin{tabular}{|l|l|l|}
\hline Themes & Occurrences & Percentage \\
\hline Customer Support Issues & 2765 & $5.7 \%$ \\
\hline Delay & 1986 & $4.0 \%$ \\
\hline Damaged Shipments & 511 & $1.0 \%$ \\
\hline Delivery Issues & 4736 & $9.7 \%$ \\
\hline Hidden Prices & 756 & $1.5 \%$ \\
\hline
\end{tabular}

\section{B. Delay}

Approximately $4 \%$ of the negative tweets showed negative attitudes toward delayed shipment. Customers complained that their shipments were either late or had never arrived. Some examples of these complaints are as follows:

"I hope the problem will be solved, two months have passed, and my shipment has not arrived yet.”

"My shipment was supposed to be shipped on August 1, but it was not shipped, and you were 10 days late for the supposed delivery date.”

\section{Damaged Shipments}

About $1 \%$ of the negative tweets were related to receiving damaged shipments. Customers complained that their shipments had been damaged, broken, opened, or expired. Here are some examples of these tweets:

"I received my shipment very late. And I received it with damaged products, spilled products, and the shipment arrived in a very bad condition ... Who will compensate me for the damage?”

"65 days delay and the shipment arrived opened and dirty."

"I inform you that my shipment arrived after delaying three days of calling today at $1 \mathrm{pm}$, the temperature was 47 degrees Celsius. The car was not air-conditioned, and the box was at a high temperature, noting that the shipment contains vitamins, shampoo and makeup that does not bear the heat, and it has been spoiled."

\section{Delivery Issues}

About $9.7 \%$ of the data showed negative opinions about delivery issues. Customers mentioned that they faced many issues with the delivery driver while delivering their shipment. Many customers complained that the delivery drivers were unreachable. Some examples of customer's complaints are as follow:

"A company like you should employ respectable employees who know how to deal with customer ... unfortunately your delivery driver was very bad and disrespectful today. He calls and raises his voice and talks with impoliteness to deliver the shipment! It is your duty to deliver the shipments to their owners!!”

"Respond to me first and find my shipment!! My shipment has arrived two months ago, and the delivery driver did not answer my calls, and his mobile was locked."

"I shipped my shipment through express mail and paid extra money to deliver the shipment faster ... in the end I did not receive the shipment for two weeks."

Customers also complained about missing items in their shipments, lost shipments, delivering to the wrong location, and failing to provide the promised door-to-door service. See the examples below: 
"I've called and you told me that my shipment was lost, and I've contacted you 3 months ago, and I've been told that you will communicate with me, but nothing happened.”

"Unfortunately, they charge large fees for shipping shipments, and deliveries are delayed and their shipments arrive incomplete! I have a shipment about two weeks ago, and today it arrives incomplete.”

"A paid shipment arrived two weeks ago and arrived the wrong address. I contacted you 3 times to change the location and deliver it to my home, and there is no response!”

"The delivery driver left my shipment with negligence at the end of the street and not in front of the door of the house and went, which indicates the lack of integrity of the company representative!”

\section{E. Hidden Prices}

Customers were dissatisfied with shipment companies' hidden fees. Approximately $1.5 \%$ of the data showed negative attitudes toward extra charges and taxes. The following are some examples:

"Your representative asked me to pay an additional delivery fee, or he will return my shipment. I refused to pay, and he refused to deliver."

"Why pay 30 riyals for the delivery fee? I've paid for the order shipping fee earlier!”

\section{DisCUSSION AND RECOMMENDATIONS}

Based on the above results, it is clear that logistics service providers in Saudi Arabia need to improve their service quality to maintain their credibility and reputation, and to gain customers' trust. The domain of logistics services in Saudi Arabia is still immature, although significant changes have taken place in the last decade. Logistics service providers should try to gain competitive advantages over others by providing high-quality services that distinguish them from others [12].

Communication between the customer and employees is highly important during service delivery in terms of meeting customers' expectations [12]. Logistics service providers should offer a variety of customer support mechanisms, such as instant messaging with chatbots to answer frequently asked questions, change the location details, or reschedule delivery dates [30]. It is also critical to provide real-time support with customer service representatives in order to resolve customer issues [31], [32]. In addition, logistics service providers should provide temperature-controlled vehicles and dry gel packs for fragile shipments in order to avoid damage to goods [33].

Logistics service providers should make certain that customers are always kept up to date on the status of their shipments and any delays. Radio Frequency Identification (RFID) can be used by logistics service providers to provide real-time data about shipments, allowing them to know exactly where the shipment is from start to finish. RFID can improve shipment visibility, prevent shipment loss, prevent delivery to the incorrect destination, and reduce customer frustration caused by shipment delays by providing them with a real-time delivery plan. RFID also reduces the amount of effort and time required to resolve these issues [34].

To resolve issues with unclear fees, transparency should be increased by creating an informed list that shows the shipping process, information required from customers, and a detailed bill. This procedure will save time and money while also increasing customer satisfaction [35]. Additionally, to mitigate the impact of delayed shipments on customer satisfaction during peak times, logistics service providers can offer customers discount coupons as a form of compensation for the delays and to reduce their disappointment and anger [36]. With regard to delivery drivers, logistics service providers' systems should allow customers to rate their experience with the delivery drivers. This would allow the company to identify and control drivers' behavior, improve the service quality, and ensure customer satisfaction [37]. In addition, the system could increase employee loyalty by providing bonuses to the highest-ranking employees [38].

\section{CONCLUSION}

In this paper, we have evaluated the quality of logistics services during the COVID-19 pandemic in Saudi Arabia by analyzing customers' opinions. Sentiment analysis was performed to analyze customers' opinions expressed via the Twitter platform. Specifically, an SVM classifier was applied to predict the sentiment polarity of customers' opinions; this resulted in $82 \%$ accuracy, $81 \%$ precision, and $80 \%$ recall. Our findings revealed that the majority of the opinions were negative. Further investigation using thematic analysis revealed a number of themes representing factors that had a negative impact on the quality of logistics services. Based on the findings, we developed a set of recommendations to help logistics service providers improve their service quality. We intend to expand the lexicon by adding more Saudi dialects in the future, as well as broadening our approach to analyzing customer reviews on apps. This will allow us to assess companies' strengths and weaknesses and to assist them in improving the quality of their services via their apps.

\section{REFERENCES}

[1] T. P. Stank, T. J. Goldsby, and S. K. Vickery, "Logistics service performance: Estimating its influence on market share," J. Bus. Logist., vol. 24, no. 1, pp. 27-55, 2003.

[2] Bowersox, Donald J., David J. Cioss, and M. Bixby Cooper (2002), Supply Chain Logistics Management, New York: McGraw-Hill/Irwin, p. 34.

[3] I. Meidutè-Kavaliauskienè, A. Aranskis, and M. Litvinenko, "Consumer satisfaction with the quality of logistics services,” Procedia - Soc. Behav. Sci., vol. 110, pp. 330-340, 2014, doi: https://doi.org/10.1016/j.sbspro.2013.12.877.

[4] A. Sharma, D. Grewal, and M. Levy, "The customer satisfaction/logistics interface,” Journal of Business Logistics., vol. 16, no. 2, p. 1, 1995.

[5] J. J. Cronin and S. A. Taylor, "Measuring Service Quality: A Reexamination and Extension,” J. Mark., vol. 56, no. 3, pp. 55-68, Jul. 1992, https://doi: 10.1177/002224299205600304.

[6] B. Pang and L. Lee, "Opinion mining and sentiment analysis," Foundations and trends in information retrieval, vol. 2, no. 1-2, , pp. 1135, 2008.

[7] F. Buttle, "Customer relationship management: concepts and technology,” Sydney: a Butterworth-Heinemann, vol. 1, 2009.

[8] D. K. Tse, F. M. Nicosia, and P. C. Wilton, "Consumer satisfaction as a process,” Psychology \& Marketing, vol. 7, no. 3, p. 177, 1990. 
[9] A. Parasuraman, V. A. Zeithaml, and L. L. Berry, "Servqual: A multiple-item scale for measuring consumer perc,” J. Retail., vol. 64, no. 1, pp. 12-40, 1988.

[10] D. N. Le, H. T. Nguyen, and P. H. Truong, "The Asian Journal of Shipping and Logistics Port logistics service quality and customer satisfaction: Empirical evidence from Vietnam,” Asian J. Shipp. Logist., vol. 36, no. 2, pp. 89-103, 2020, doi: 10.1016/j.ajsl.2019.10.003.

[11] C.-H. Cho, B.-I. Kim, and J.-H. Hyun, "A comparative analysis of the ports of Incheon and Shanghai: The cognitive service quality of ports, customer satisfaction, and post-behaviour," Total Qual. Manag. Bus. Excell., vol. 21, no. 9, pp. 919-930, Sep. 2010, doi: 10.1080/14783363.2010.487677.

[12] H. Uvet, "Importance of logistics service quality in customer satisfaction: An empirical study,” Oper. Supply Chain Manag. An Int. J., vol. 13, no. 1, pp. 1-10, 2020.

[13] S. Anastasia and I. Budi, "Twitter sentiment analysis of online transportation service providers," in 2016 International Conference on Advanced Computer Science and Information Systems, ICACSIS 2016, pp. 359-365, doi: 10.1109/ICACSIS.2016.7872807.

[14] S. Gitto and P. Mancuso, "Improving airport services using sentiment analysis of the websites,” Tour. Manag. Perspect., vol. 22, pp. 132-136, Apr. 2017, doi: 10.1016/j.tmp.2017.03.008.

[15] J. Bhattacharjya, A. Ellison, and S. Tripathi, "An exploration of logistics-related customer service provision on Twitter: The case of eretailers,” Int. J. Phys. Distrib. Logist. Manag., vol. 46, no. 6-7, pp. 659-680, Jul. 2016, doi: 10.1108/IJPDLM-01-2015-0007.

[16] S. Ahmadi, S. Shokouhyar, M. H. Shahidzadeh, and I. Elpiniki Papageorgiou, "The bright side of consumers' opinions of improving reverse logistics decisions: A social media analytic framework," International Journal of Logistics Research and Applications., 2020, doi: 10.1080/13675567.2020.1846693.

[17] H. K. Aldayel and A. M. Azmi, "Arabic tweets sentiment analysis - A hybrid scheme,” J. Inf. Sci., vol. 42, no. 6, pp. 782-797, Dec. 2016, doi: 10.1177/0165551515610513.

[18] W. Al-Ghaith, "Developing lexicon-based algorithms and sentiment lexicon for sentiment analysis of Saudi dialect tweets," International Journal of Advanced Computer Science and Applications(IJACSA), 10(11), 2019. http://dx.doi.org/10.14569/IJACSA.2019.0101112.

[19] L. Al-Horaibi and M. B. Khan, "Sentiment Analysis of Arabic Tweets Using Semantic Resources,” Int. J. Comput. Inf. Sci., vol. 12, no. 2, 2016, doi: 10.21700/ijcis.2016.118.

[20] H. Al-Hussaini and H. Al-Dossari, "A Lexicon-based Approach to Build Service Provider Reputation from Arabic Tweets in Twitter" International Journal of Advanced Computer Science and Applications(IJACSA), $\quad$ 8(4), 2017. http://dx.doi.org/10.14569/IJACSA.2017.080459.

[21] K. Taghva, R. Elkhoury, and J. Coombs, "Arabic stemming without a root dictionary,” Int. Conf. Inf. Technol. Coding Comput. - Vol. II, vol. 1, pp. 152-157, 2005, doi: 10.1109/ITCC.2005.90.

[22] L. Albraheem and H. S. Al-Khalifa, "Exploring the problems of sentiment analysis in informal Arabic," in ACM International Conference Proceeding Series, 2012, pp. 415-418, doi: 10.1145/2428736.2428813.

[23] A. Assiri, A. Emam, and H. Aldossari, "Arabic Sentiment Analysis: A Survey,” International Journal of Advanced Computer Science and Applications(IJACSA)， 6(12)， $2015 . \quad$ http://dx.doi.org/10.14569/ IJACSA.2015.061211.
[24] S. Almujaiwel, “Covid-19_1M_Saudi_Tweets. GitHub repository,” 2020. https://github.com/salmujaiwel/Covid-19_1M_Saudi_Tweets (accessed Jun. 04, 2021).

[25] N. Alrumayyan, S. Bawazeer, R. AlJurayyad, and M. Al-Razgan, "Analyzing user behaviors: A study of tips in foursquare," in Advances in Intelligent Systems and Computing, vol. 753, pp. 153-168, 2018. doi: 10.1007/978-3-319-78753-4_12.

[26] N. Cristianini and B. Scholkopf, "Support vector machines and kernel methods: The new generation of learning machines," AI Mag., vol. 23, no. 3 SE-Articles, p. 31, Sep. 2002, doi: 10.1609/aimag.v23i3.1655.

[27] G. Fung and O. L. Mangasarian, "Incremental support vector machine classification." In Proceedings of the 2002 SIAM International Conference on Data Mining, pp. 247-260. Society for Industrial and Applied Mathematics, 2002.

[28] R. M. Duwairi and I. Qarqaz, "Arabic sentiment analysis using supervised classification,” in Proceedings - 2014 International Conference on Future Internet of Things and Cloud, FiCloud 2014, Dec. 2014, pp. 579-583, doi: 10.1109/FiCloud.2014.100.

[29] W. Alabbas, H. M. Al-Khateeb, A. Mansour, G. Epiphaniou, and I. Frommholz, "Classification of colloquial Arabic tweets in real-time to detect high-risk floods," in 2017 International Conference On Social Media, Wearable And Web Analytics, Social Media 2017, Oct. 2017, vol. 2017-June, pp. 1-8, doi: 10.1109/SOCIALMEDIA.2017.8057358.

[30] V. Marino and L. Lo Presti, "Engagement, satisfaction and customer behavior-based CRM performance,” J. Serv. Theory Pract., vol. 28, no. 5, pp. 682-707, Jan. 2018, doi: 10.1108/JSTP-11-2017-0222.

[31] Y. Park and S. C. Gates, "Towards real-time measurement of customer satisfaction using automatically generated call transcripts," in Proceedings of the 18th ACM conference on Information and knowledge management, 2009, pp. 1387-1396, doi: $10.1145 / 1645953.1646128$.

[32] B. J. Goldenberg, CRM in real time: empowering customer relationships. Information Today, Inc., 2008, pp.293.

[33] C. Sykes, "Time- and temperature-controlled transport: Supply chain challenges and solutions,” P\& T: a peer-reviewed journal for formulary management, vol. 43, no. 3, 2018, pp. 154-170.

[34] S. Garrido Azevedo and H. Carvalho, "Contribution of RFID technology to better management of fashion supply chains," Int. J. Retail Distrib. Manag., vol. 40, no. 2, pp. 128-156, Jan. 2012, doi: 10.1108/09590551211201874.

[35] Z. Tian, R. Y. Zhong, A. Vatankhah Barenji, Y. T. Wang, Z. Li, and Y. Rong, "A blockchain-based evaluation approach for customer delivery satisfaction in sustainable urban logistics,” Int. J. Prod. Res., vol. 59, no. 7, pp. 2229-2249, 2021.

[36] D.-S. Chang and T.-H. Wang, "Consumer preferences for service recovery options after delivery delay when shopping online," Soc. Behav. Personal. an Int. J., vol. 40, no. 6, pp. 1033-1043, 2012.

[37] F. Xiang and W. Wu, "Research on emotional labor and management of takeaway delivery staff," in 6th Annual International Conference on Social Science and Contemporary Humanity Development (SSCHD 2020), 2021, pp. 485-489.

[38] S. Moro, R. F. Ramos, and P. Rita, "What drives job satisfaction in IT companies?,” International Journal of Productivity and Performance Management, vol. 70, no. 2, pp. 391-407, 2021, https://doi.org/10.1108/IJPPM-03-2019-0124. 